\title{
COVID-19: Tendências em mudança e seu impacto no futuro da odontologia.
}

Antônio Athayde (1) e Manoel Fernando Silva (1)

\section{ARTIGO ORIGINAL}

Resumo

O surto da doença coronavírus 2019 (COVID-19) rapidamente se transformou em uma pandemia mundial, criando uma crise econômica e de saúde global. É um vírus novo, distinto do SARS-CoV e do MERS-CoV, sendo o morcego-ferradura chinês a origem mais provável. A transmissão ocorre principalmente através da propagação de gotículas ou rotas de contato. Devido às características dos ambientes odontológicos, o risco de infecção cruzada entre o pessoal de saúde odontológica (DHCP) e os pacientes pode ser muito alto. Este artigo fornece uma breve visão geral da estrutura do vírus, modos de transmissão e características clínicas da doença COVID-19. O objetivo deste artigo é recomendar estratégias de controle de infecção e protocolos de manejo de pacientes para fornecer atendimento odontológico ideal e, simultaneamente, prevenir infecção hospitalar em ambientes odontológicos.

Palavras Cheave: Covid-19, Odontologia, Sintomas, Saúde bucal. 


\title{
COVID-19: Changing trends and their impact on the future of dentistry.
}

\begin{abstract}
The 2019 coronavirus outbreak (COVID-19) quickly turned into a worldwide pandemic, creating a global economic and health crisis. It is a new virus, distinct from SARS-CoV and MERS-CoV, with the Chinese horseshoe bat being the most likely source. Transmission occurs mainly through the propagation of droplets or contact routes. Due to the characteristics of dental environments, the risk of cross-infection between dental health personnel (DHCP) and patients can be very high. This article provides a brief overview of the virus structure, modes of transmission and clinical characteristics of COVID-19 disease. The purpose of this article is to recommend infection control strategies and patient management protocols to provide optimal dental care and, at the same time, prevent hospital infection in dental environments.
\end{abstract}

Key words: Covid-19, Dentistry, Symptoms, Oral health.

Instituição afiliada: 1- Professor de Diagnóstico I e II do Centro Universitário do Pará.

Dados da publicação: Artigo recebido em 10 de Fevereiro, revisado em 15 de Fevereiro, aceito para publicação em 20 de Fevereiro e publicado em 29 de Fevereiro.

DOI: DOI: $\underline{\text { https://doi.org/10.36557/2674-8169.2021v3n2p11-23 }}$

Antonio Athayde athaydebicolor@gmail.com

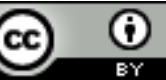

This work is licensed under a Creative Commons Attribution 4.0 International

License. 


\section{INTRODUÇÃO}

Várias epidemias (como H1N1, H5N1, gripe aviária, Ebola, SARS, Zika e Nipah) afetaram a Índia e outros países no passado, que foram enfrentados com sucesso com pesquisas apropriadas [1]. O surgimento de novos patógenos humanos e o ressurgimento de várias doenças são de particular preocupação [2]. Um novo coronavírus humano inicialmente referido como coronavírus Wuhan (CoV), atualmente designado como síndrome respiratória aguda grave (SARS) -CoV-2, é responsável pela última pandemia que está afetando a saúde humana e a economia em todo o mundo [ 3] Em 30 de janeiro de 2020, a OMS declarou o surto chinês de COVID-19 como uma Emergência de Saúde Pública de Preocupação Internacional por causa de sua disseminação desenfreada, representando assim um alto risco para países com sistemas de saúde vulneráveis. De acordo com a atualização do relatório de situação da OMS (14 de maio de 2020) no COVID-19, houve mais de 42,48,389 casos notificados e 2,94,046 mortes em todo o mundo [ 4 ]. Ao impor um bloqueio em todo o país, a Índia reduziu a disseminação desse vírus até certo ponto; entretanto, o número total de casos relatados ultrapassou 78.000 com aproximadamente 2.500 mortes e esses números continuam a aumentar [ 4 ].

Dada a ampla transmissão do SARS-CoV-2, os profissionais de saúde correm um risco maior de contrair a infecção e se tornarem potenciais portadores da doença. De acordo com a Administração de Segurança e Saúde Ocupacional (OSHA), o pessoal de saúde bucal (PDHC) é colocado na categoria de risco de exposição muito alto, pois os dentistas trabalham próximos à cavidade oral do paciente [5]. Além disso, os procedimentos odontológicos envolvem o uso de instrumentos rotativos, como peças de mão e raspadores, que geram aerossóis. Assim, é necessária uma maior compreensão da estrutura do vírus, modos de transmissão, características clínicas e métodos de teste que podem ajudar a formar protocolos para consultórios odontológicos para identificar casos e prevenir a propagação da infecção para os pacientes e DHCP.

\section{Estrutura}

O SARS-CoV-2 é o sétimo membro da família dos coronavírus que infectam humanos. Embora semelhante a alguns betacoronavírus, é distinto do SARS-CoV e do MERS-CoV. É um novo vírus pertencente ao subgênero sarbecovirus, subfamília Orthocoronavirinae, sendo o morcego-ferradura chinês ( Rhinolophus sinicus) a origem mais provável [ 6 ]. É um vírus de RNA de fita positiva com envelope com diâmetro de 60-140 nm, forma esférica ou elíptica e pleomórfica que mostra uma aparência semelhante a uma coroa sob um microscópio eletrônico (coronam é o termo latino para coroa) $[7,8]$

\section{Manifestações clínicas}

Os sintomas comuns no início da doença incluem febre, tosse não produtiva, mialgia ou fadiga; os sintomas menos comuns são produção de expectoração, cefaleia, 
hemoptise e diarreia [9]. Outro sintoma comum é a pneumonia, que pode ser observada na radiografia de tórax ou na tomografia computadorizada de tórax como várias pequenas sombras irregulares e alterações intersticiais, notáveis na periferia do pulmão. Disfunções de órgãos, como síndrome do desconforto respiratório agudo (SDRA), lesão cardíaca aguda (choque e arritmia), lesão renal aguda e morte podem ocorrer em casos graves [ 9,10 ]. Idade e comorbidade foram considerados fatores de risco para resultados ruins [ 10$]$.

\section{Tratamento / Gestão}

O manejo clínico de COVID-19 é principalmente o tratamento sintomático. Os casos graves requerem assistência respiratória com suporte de órgãos em terapia intensiva. Não existe nenhum tratamento antiviral específico, mas drogas antivirais, antimaláricas e biológicas são administradas em ensaios clínicos [ 11 ].

\section{Transmissão}

Embora a transmissão animal para humano fosse presumida como o principal mecanismo, agora se percebeu que os pacientes infectados, sejam sintomáticos ou assintomáticos, são as principais fontes de transmissão da infecção [ 10 ]. As evidências atuais sugerem que a transmissão de pessoa para pessoa ocorre principalmente por meio da propagação de gotículas ou de rotas de contato. A transmissão via gotículas ocorre apenas em casos de contato próximo (dentro de 1 metro) com aqueles que têm sintomas respiratórios, pois há risco de exposição da mucosa oral / nasal ou conjuntiva à exposição potencial de gotículas respiratórias infectadas quando a pessoa espirra, tosse ou fala alto [ 12 ].

Em uma configuração odontológica, além de gotículas, procedimentos em pacientes odontológicos envolvendo o uso de peças de mão de alta velocidade ou instrumentos ultrassônicos podem fazer com que suas secreções, saliva ou sangue aerosolizem o vírus nas proximidades. Assim, a transmissão também pode ocorrer por contato indireto, tocando em superfícies contaminadas, seguido de autotransmissão aos olhos, nariz ou boca [ 13 ]. As medidas de controle de infecção padrão que são seguidas no trabalho clínico diário, portanto, não serão eficazes o suficiente para prevenir a disseminação de COVID-19, especialmente quando os pacientes estão no período de incubação, não sabem que estão infectados ou optam por ocultar sua infecção [ 6 ].

\section{Configuração odontológica}

O DHCP (dentistas, higienistas dentais, assistentes dentários e recepcionistas) precisa atualizar seus conhecimentos e habilidades em relação ao controle de infecções e seguir os protocolos recomendados pelas autoridades competentes para proteger a si e seus pacientes contra infecções.

Deve-se tentar fazer a triagem por telefone de todos os pacientes que precisam de atendimento odontológico. A teledontologia pode ser de grande ajuda na atual situação de pandemia. As novas tecnologias não só melhoraram a qualidade do tratamento de pacientes odontológicos, mas também tornaram possível seu 
gerenciamento parcial ou total a distâncias de quilômetros de centros de saúde ou clínicas odontológicas. Todo o processo de networking, compartilhamento de informações digitais, consultas à distância, workup e análise é tratado por um segmento da ciência da telemedicina voltado para a odontologia conhecido como "Teledentistry" $[14,15]$.

Com base nos sinais e sintomas dos pacientes, deve-se tomar uma decisão para determinar se o paciente precisa ser visto na clínica odontológica. Produtos farmacêuticos apropriados e instruções detalhadas sobre cuidados domiciliares devem ser fornecidos por meio de Teledentistry em situações onde o tratamento odontológico pode ser adiado [ 5 ].

Com base nas informações fornecidas pela American Dental Association (ADA), elaboramos uma tabela que ajuda a decidir o que constitui uma emergência odontológica; entretanto, os dentistas devem usar seu julgamento profissional para determinar a necessidade de um paciente para atendimento de urgência ou emergência (Tabela 1 ) [ 16 ].

Tabela 1: O que constitui uma emergência dentária? (adaptado da American Dental Association [ 15 ]).

\begin{tabular}{|c|c|c|}
\hline Emergências dentárias & Atendimento odontológico urgente & Outro atendimento odontológico urgente \\
\hline (i) Sangramento descontrolado & (i) Dor de dente severa por inflamação pulpar & $\begin{array}{l}\text { (i) Cárie dentária extensa ou restauraçōes defeituosas causando } \\
\text { dor }\end{array}$ \\
\hline $\begin{array}{l}\text { (ii) Celulite ou uma infecção bacteriana difusa de tecidos moles com edema intra } \\
\text { ou extraoral que potencialmente compromete as vias aéreas do paciente }\end{array}$ & (ii) Pericoronite ou dor no terceiro molar & $\begin{array}{l}\text { (ii) Gerenciar com técnicas restauradoras provisórias, quando } \\
\text { possível (fluoreto de diamina de prata, ionômeros de vidro) }\end{array}$ \\
\hline \multirow[t]{8}{*}{$\begin{array}{l}\text { (iii) Trauma envolvendo ossos faciais, potencialmente comprometendo as vias } \\
\text { aéreas do paciente }\end{array}$} & $\begin{array}{l}\text { (iii) Osteíte pós-operatória cirúrgica, trocas de curativo de } \\
\text { alvéolo seco }\end{array}$ & (iii) Remoção de sutura \\
\hline & $\begin{array}{l}\text { (iv) Abscesso ou infecção bacteriana localizada, resultando } \\
\text { em dor localizada e inchaço }\end{array}$ & (iv) Ajuste de prótese em pacientes com radiação / oncologia \\
\hline & $\begin{array}{l}\text { (v) Fratura do dente resultando em dor ou causando trauma } \\
\text { nos tecidos moles }\end{array}$ & $\begin{array}{l}\text { (v) Ajustes ou reparos de dentadura quando a função é } \\
\text { impedida }\end{array}$ \\
\hline & (vi) Trauma dentário com avulsão / luxação & $\begin{array}{l}\text { (vi) Substituição do enchimento temporário em aberturas de } \\
\text { endo-acesso em pacientes com dor }\end{array}$ \\
\hline & $\begin{array}{l}\text { (vii) Tratamento dentário necessário antes de procedimentos } \\
\text { médicos críticos }\end{array}$ & $\begin{array}{l}\text { (vii) Corte ou ajuste de um fio ortodôntico ou aparelhos } \\
\text { perfurando ou ulcerando a mucosa oral }\end{array}$ \\
\hline & (viii) Cimentação final da coroa / ponte se a restauração & \\
\hline & temporária for perdida, quebrada ou causar irritação gengival & \\
\hline & (ix) Biópsia de tecido anormal & \\
\hline
\end{tabular}

Após a decisão de que o paciente precisa visitar a clínica odontológica, a próxima etapa deve ser avaliar os pacientes quanto a sinais e sintomas de infecção por COVID-19 para determinar em qual ambiente clínico eles devem ser examinados. De acordo com as orientações do Centro de Controle e Prevenção de Doenças (CDC), os pacientes com infecção COVID-19 ativa não devem ser vistos em ambientes odontológicos e devem ser encaminhados para atendimento de emergência onde as precauções baseadas na transmissão apropriadas estiverem disponíveis [ 17 ].

Uma história detalhada deve ser obtida dos pacientes solicitando-lhes o preenchimento do formulário de triagem para infecção por COVID-19, que deve incluir as seguintes perguntas: (1) Você tem febre ou teve febre nos últimos 14 dias? (2) Você 
teve um início recente de problemas respiratórios, como tosse ou dificuldade em respirar, nos últimos 14 dias? (3) Você, nos últimos 14 dias, viajou para países com transmissão documentada (SARS) -CoV-2? Ou você já teve contato com pessoas que viajaram para esses países? (4) Você entrou em contato com um paciente com infecção confirmada (SARS) -CoV-2 nos últimos 14 dias? (5) Há pessoas com experiência documentada de febre ou problemas respiratórios nos últimos 14 dias que tiveram contato próximo com você? (7) Você recentemente participou de alguma reunião, reuniões, ou teve contato próximo com muitas pessoas desconhecidas? [18 ].

Após a chegada do paciente, a temperatura corporal do paciente deve ser medida usando um termômetro frontal sem contato. Se o paciente responder "não" a todas as questões e se o paciente estiver afebril, o paciente pode ser tratado pelo cirurgião-dentista seguindo os protocolos recomendados (Figura 1 ). A capacidade de testar os pacientes que precisam de atendimento odontológico para SARS-CoV-2 deve ser considerada para reiniciar a odontologia de forma sustentável. Os testes podem ser uma ferramenta forte para mitigar os riscos para pacientes e profissionais de saúde bucal [ 19].

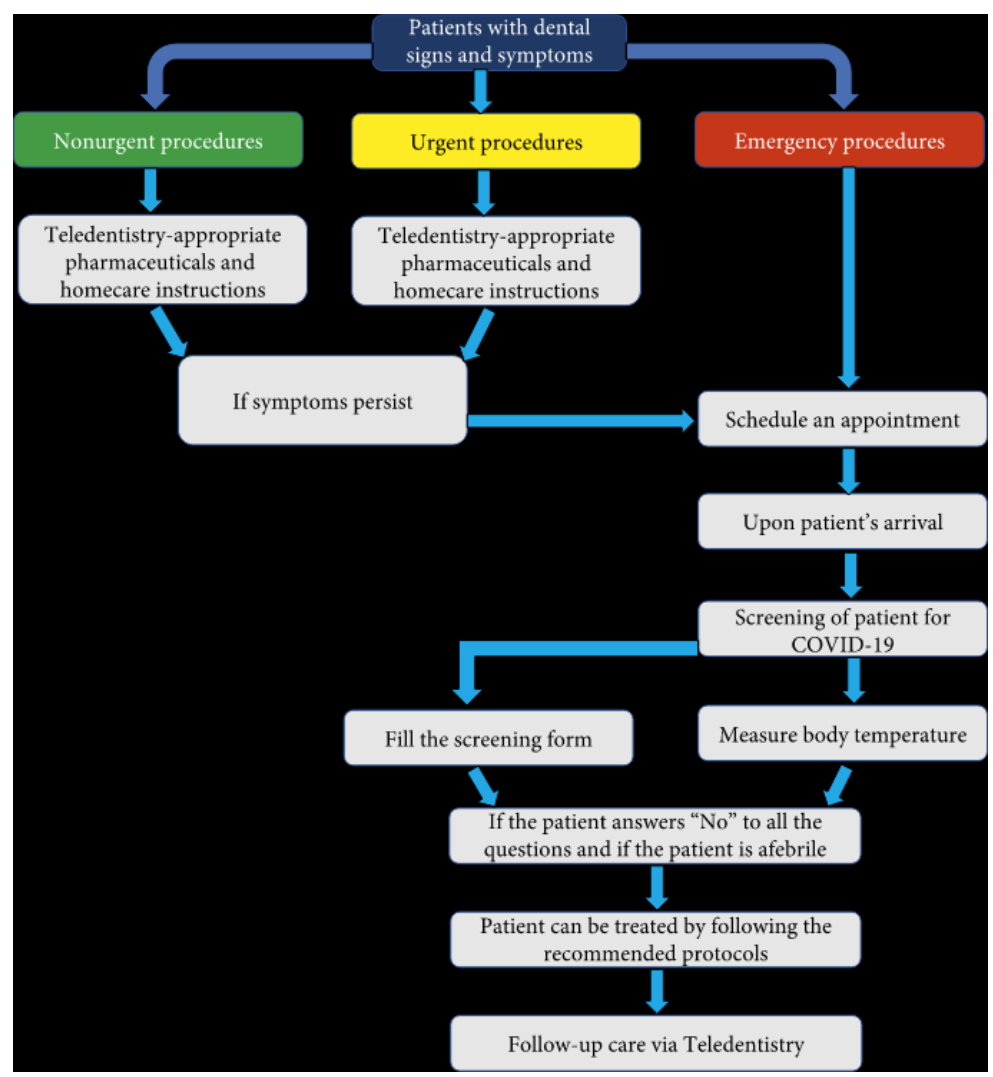

Figura 1: Gerenciamento de problemas dentários durante a pandemia de COVID19.

\section{Sala de espera}

A Indian Dental Association recomenda a postagem de ícones de alerta visual como placas e cartazes na entrada e em locais estratégicos para fornecer aos pacientes instruções (em idiomas apropriados) sobre higiene das mãos, higiene respiratória e etiqueta para tosse. As instruções devem incluir como usar lenços para cobrir o nariz e a 
boca ao tossir ou espirrar e descartar lenços e itens contaminados em recipientes de lixo e como e quando realizar a higiene das mãos [ 20 ].

As consultas devem ser agendadas de forma que o distanciamento social possa ser mantido na sala de espera. Outra alternativa é o paciente aguardar fora ou no veículo e ser contatado por telefone quando for a sua vez de ser atendido. Recomendase que o paciente evite trazer acompanhantes para a consulta, exceto nos casos em que o paciente necessite de assistência. Isso pode ser comunicado ao paciente no momento do agendamento de uma consulta [ 17 ].

\section{Durante o tratamento}

A Força Tarefa Nacional para Covid-19, constituída pelo Conselho Indiano de Pesquisa Médica, recomenda o uso de hidroxicloroquina para profilaxia da infecção por SARS-Cov-2 para profissionais de saúde envolvidos no atendimento de casos suspeitos ou confirmados de COVID-19. A dosagem recomendada é de $400 \mathrm{mg}$ duas vezes ao dia no dia 1, seguido de $400 \mathrm{mg}$ uma vez por semana durante as sete semanas seguintes, para ser tomado com as refeições [ 21 ].

Recomenda-se que o mais alto nível de equipamento de proteção individual (EPI) disponível seja usado pelo cirurgião-dentista e assistente dentário durante o tratamento de pacientes, o que inclui luvas, jaleco, proteção para a cabeça, proteção para sapatos, proteção para os olhos, incluindo óculos ou uma máscara facial descartável / reutilizável que cobre a frente e os lados do rosto e um respirador N954 ou de nível superior. Uma combinação de máscara cirúrgica e protetor facial pode ser usada em situações em que o respirador não está disponível [ 13 ].

Uma boa higiene das mãos é uma das melhores maneiras de prevenir a propagação de doenças infecciosas. Deve-se seguir uma higienização das mãos dois antes e três depois, a fim de reforçar a conformidade da lavagem das mãos. Especificamente, o cirurgião-dentista e o assistente de dentista devem lavar as mãos antes de examinar um paciente, antes de realizar qualquer procedimento odontológico, após tocar o paciente, após tocar os arredores e equipamentos sem desinfecção, e após tocar a mucosa oral, sangue, pele danificada, ou ferida [ 18 ].

Enxaguatório bucal pré-procedimento com 0,5-1\% de peróxido de hidrogênio por sua atividade virucida não específica contra vírus ou com iodo-povidona a 0,2\% é recomendado, pois pode reduzir a carga do vírus corona na saliva [ 22 ].

$O$ exame de raios- $X$ intraoral é a técnica radiográfica mais comum em imagens dentárias; no entanto, pode estimular a secreção de saliva e a tosse. Portanto, radiografias dentárias extraorais, como a radiografia panorâmica e a tomografia computadorizada de feixe cônico (TCFC), são alternativas adequadas durante o surto de COVID-19 [ 6 ].

O DHCP deve evitar da melhor forma os procedimentos de geração de aerossol e priorizar o uso de instrumentos manuais, como escavadeiras de colher, em combinação com agentes de remoção de cárie quimomecânicos. No entanto, se for necessário realizar um procedimento gerador de aerossol, ele deve ser agendado como a última 
consulta do dia [18]. Recomenda-se trabalhar a partir da posição de 10 ou 11 horas. Para evitar respingos, a posição das oito horas deve ser evitada. $O$ uso de barragem de borracha durante esses procedimentos é recomendado, pois pode reduzir significativamente as partículas transportadas pelo ar em aproximadamente um metro de diâmetro do campo operacional em $70 \%$. Odontologia a quatro mãos com sucção de alto volume para aerossóis deve ser implementada junto com a suç̧ão regular [18] Medidas adicionais, como melhorar a qualidade da água, enxágue das linhas d'água da unidade odontológica, uso de válvulas anti-retração, peças de mão anti-retração e aspiração retrógrada são fortemente recomendadas para prevenir infecções cruzadas [23].

\section{Pós-tratamento}

Como os coronavírus perdem sua viabilidade significativamente após 72 horas, muitas organizações promoveram uma estratégia de rotação e reutilização. Envolve a aquisição de um determinado número de máscaras N95 (pelo menos 5 de acordo com o CDC) e alterna seu uso a cada dia, permitindo que sequem por tempo suficiente para que o vírus não seja mais viável [ 24 ]. No entanto, respiradores N95 usados durante procedimentos geradores de aerossol ou aqueles contaminados com sangue, secreções respiratórias ou nasais ou outros fluidos corporais de pacientes devem ser descartados [25].

A fumigação não é prática para cirurgias odontológicas; no entanto, medidas como limpar o chão com hipoclorito de sódio a $1 \%$ e desinfetar linhas de água com hipoclorito de sódio a $0,01 \%$ podem ajudar a reduzir o risco de infecção cruzada [26]. Todos os resíduos biomédicos relativos ao cuidado do paciente devem ser descartados cuidadosamente de vez em quando por meio de uma agência autorizada de descarte biomédico [ 27 ].

A teledontologia como uma forma de telessaúde fornece uma abordagem pragmática para avaliar e registrar o estado de saúde bucal no pós-operatório e, portanto, melhorar a prestação geral de cuidados bucais [28] O dentista pode monitorar os resultados do tratamento por meio de fotografia móvel garantindo a confidencialidade do paciente e também fornecer vídeos educacionais sobre a manutenção da higiene bucal para o benefício do paciente. Com uma mudança de paradigma na prática de atendimento odontológico em curso durante a atual situação de pandemia, a Teledentistry traz a perspectiva de atender às necessidades de tratamento dos pacientes sem confronto. Não apenas elimina qualquer chance de exposição ao vírus, mas também diminui o custo do serviço e ajuda na educação do paciente e, mais importante, o distanciamento social pode ser mantido. A teledontologia mudou a perspectiva da odontologia e nunca ganhou um ponto de apoio mais forte na prática, como provavelmente aconteceu nessa época. Portanto, é fundamental que o DHCP abrace essa ferramenta fundamental e a aplique em todo o seu potencial. 


\section{CONCLUSÃO}

Os profissionais de saúde bucal precisam entender as implicações da transmissão potencial do vírus (SARS) -CoV-2 em um ambiente clínico. Por isso, eles precisam se manter atualizados com as novas informações a respeito dessa doença. Novas abordagens, como a teledentística, ajudarão os dentistas a auxiliar os pacientes sem aumentar o risco de infecção cruzada. A recente situação impõe a necessidade de encontrar um equilíbrio entre a segurança dos profissionais de saúde e ainda fornecer atendimento odontológico ideal aos pacientes que requerem intervenção de emergência.

\section{OS AUTORES DECLARAM NÃO HAVER CONFLITOS DE INTERESSE}

\section{REFERÊNCIAS}

1. R. Mathur, "Ethics preparedness for infectious disease outbreaks research in India: a case for novel coronavirus disease 2019," The Indian Journal of Medical Research, vol. 151, no. 3, pp. 124-131, 2020.View at: Publisher Site | Google Scholar

2. D. T. Mourya, P. D. Yadav, P. T. Ullas et al., "Emerging/re-emerging viral diseases \& new viruses on the Indian horizon," Indian Journal of Medical Research, vol. 149, pp. 447467, 2017.View at: Publisher Site | Google Scholar

3. S. Prasad, V. Potdar, S. Cherian, P. Abraham, and A. Basu, "Transmission electron microscopy imaging of SARS-CoV-2," Indian Journal of Medical Research, vol. 151, no. 3, p. 243, 2020.View at: Publisher Site | Google Scholar

4. World Health Organization, Coronavirus Disease 2019 (COVID-19) Situation Report-97, World Health Organization, Geneva, Switzerland, 2019, https://www.who.int/docs/default-source/coronaviruse/situation-reports/20200514covid-19-sitrep-115.pdf?sfvrsn=3fce8d3c_4.

5. Centers for Disease Control and Prevention, Interim Infection Prevention and Control Guidance for Dental Settings during the COVID-19 Response, Centers for Disease Control and Prevention, Atlanta, GA, USA, 2019, https://www.cdc.gov/coronavirus/2019-ncov/hcp/dental-settings.html.

6. L. Meng, F. Hua, and Z. Bian, "Coronavirus disease 2019 (COVID-19): emerging and future challenges for dental and oral Medicine," Journal of Dental Research, vol. 99, 
no. 5, pp. 481-487, 2020.View at: Publisher Site | Google Scholar

7. Z. Chen, J. Fu, Q. Shu et al., "Diagnosis and treatment recommendations for pediatric respiratory infection caused by the 2019 novel coronavirus," World Journal of Pediatrics, pp. 1-7, 2020.View at: Publisher Site | Google Scholar

8. N. Zhu, D. Zhang, W. Wang et al., "A novel coronavirus from patients with pneumonia in China, 2019," New England Journal of Medicine, vol. 382, no. 8, pp. 727-733, 2020.View at: Publisher Site | Google Scholar

9. C. Huang, Y. Wang, X. Li et al., "Clinical features of patients infected with 2019 novel coronavirus in Wuhan, China," The Lancet, vol. 395, no. 10223, pp. 497-506, 2020.View at: Publisher Site | Google Scholar

10. J. F.-W. Chan, S. Yuan, K.-H. Kok et al., "A familial cluster of pneumonia associated with the 2019 novel coronavirus indicating person-to-person transmission: a study of a family cluster," The Lancet, vol. 395, no. 10223, pp. 514-523, 2020.View at: Publisher Site | Google Scholar

11. F. Bennardo, C. Buffone, and A. Giudice, "New therapeutic opportunities for COVID-19 patients with Tocilizumab: possible correlation of interleukin-6 receptor inhibitors with osteonecrosis of the jaws," Oral Oncology, Article ID 104659, 2020.View at: Publisher Site | Google Scholar

12. World Health Organization, Modes of Transmission of Virus Causing COVID-19: Implications for IPC Precaution Recommendations, World Health Organization, Geneva, Switzerland, 2019, https://www.who.int/news$\mathrm{room} /$ commentaries/detail/modes-of-transmission-of-virus-causing-covid-19implications-for-ipc-precaution-recommendations.

13. Centers for Disease Control and Prevention, Interim Infection Prevention and Control Recommendations for Patients with Suspected or Confirmed Coronavirus Disease 2019 (COVID-19) in Healthcare Settings, Centers for Disease Control and Prevention, Atlanta, GA, USA, 2019, https://www.cdc.gov/coronavirus/2019-ncov/hcp/infection-controlrecommendations.html.

14. N. D. Jampani, R. Nutalapati, B. S. K. Dontula, and R. Boyapati, "Applications of teledentistry: a literature review and update," Journal of International Society of Preventive and Community Dentistry, vol. 1, no. 2, pp. 37-44, 2011.View at: Publisher Site | Google Scholar

15. B. Mihailovic, M. Miladinovic, and B. Vujicic, "Telemedicine in dentistry (teledentistry)," in Advances in Telemedicine: Applications in Various Medical Disciplines and Geographical Areas 2011, G. Graschew and T. A. Roelofs, Eds., pp. 215-230, InTech, Rijeka, Croatia, 2011.View at: Publisher Site | Google Scholar 
16. ADA, What Constitutes a Dental Emergency? American Dental Association, Chicago, IL, USA, 2020, https://success.ada.org/ /media/CPS/Files/Open/Files/ADA_COVID19_Dental_Emerg ency_DDS.pdf?_ga=2.192749678.532705002.1587280727-1559243850.1586521900.

17. ADA, Interim Guidance for Minimizing Risk of COVID-19 Transmission, American Dental Association, Chicago, IL, USA, 2020, https://www.ada.org/interimguidance.

18. X. Peng, X. Xu, Y. Li, L. Cheng, X. Zhou, and B. Ren, "Transmission routes of 2019-nCoV and controls in dental practice," International Journal of Oral Science, vol. 12, no. 1, pp. 1-6, 2020.View at: Publisher Site | Google Scholar

19. A. Giudice, A. Antonelli, and F. Bennardo, "To test or not to test? An opportunity to restart dentistry sustainably in "COVID-19 era"," International Endodontic Journal, 2020.View at: Publisher Site | Google Scholar

20. Indian Dental Association, Indian Dental Association's Preventive Guidelines for Dental Professionals on the Coronavirus Threat, Indian Dental Association, Maharashtra, India, 2019,

https://www.ida.org.in/pdf/IDA_Recommendations_for_Dental_Professionals_on_the _Coronavirus_Threat.pdf.

21. Ministry of Health and Family Welfare, Government of India, Advisory on the Use of Hydroxy-Chloroquine as Prophylaxis for SARS-CoV-2 Infection, Ministry of Health and Family Welfare, Government of India, New Delhi, India, 2019, https://www.mohfw.gov.in/pdf/AdvisoryontheuseofHydroxychloroquinasprophylaxisf orSARSCoV2infection.pdf.

22. A. Ather, B. Patel, N. B. Ruparel, A. Diogenes, and K. M. Hargreaves, "Coronavirus Disease 19 (COVID-19): implications for clinical dental care," Journal of Endodontics, vol. 46, no. 5, pp. 584-595, 2020.View at: Publisher Site | Google Scholar

23. Coelho Paraguassu, E. . (2020). COVID-19, a relação direta entre o capital, solidariedade e as vidas. Brazilian Journal of Implantology and Health Sciences , 2(3), 01-04. Recuperado de https://bjihs.emnuvens.com.br/bjihs/article/view/33

24. Coelho Paraguassu, E. ., Celis de Cardenas, A. M. ., Ferreira de Andrade, R. ., La Vecchia, C. ., \& Naldi, L. . (2020). The Covid-19 explosion in the state of Amapá: how is the most preserved region in the Brazilian Amazon currently fighting the SARS-COV 2 pandemic?. Brazilian Journal of Implantology and Health Sciences, 2(5), 3-11. https://doi.org/10.36557/2674-8169.2020v2n5p3-11

25. Centers for Disease Control and Prevention, Recommended Guidance for Extended Use and Limited Reuse of N95 Filtering Facepiece Respirators in Healthcare Settings, Centers for Disease Control and Prevention, Atlanta, GA, USA, 2020, 
https://www.cdc.gov/niosh/topics/hcwcontrols/recommendedguidanceextuse.html.

26. Centers for Disease Control and Prevention, Cleaning and Disinfection for Community Facilities, Centers for Disease Control and Prevention, Atlanta, GA, USA, 2020, https://www.cdc.gov/coronavirus/2019-ncov/community/organizations/cleaningdisinfection.html.

27. Dental Council of India, Covid-19 Guidelines for Dental Colleges, Dental Students and Dental Professionals by Dental Council of India, Dental Council of India, New Delhi, India, 2020, http://dciindia.gov.in/Admin/NewsArchives/DCI/Guidelines/on/COVID19.pdf.

28. Coelho Paraguassu, E. ., Chen, H. ., Zhou, F. ., Xu , Z. ., \& Wang, M. . (2020). Coronavirus and COVID-19: The latest news and views from the scientific community about the new coronavirus and COVID-19. Brazilian Journal of Implantology and Health Sciences , 2(3), 96-109. https://doi.org/10.36557/2674-8169.2020v2n3p96-109 